\title{
Usefulness of barrier function index based on water content and transepidermal water loss for evaluating efficacy of skin protective creams
}

\author{
Takahiro Kubota*, Yukina Watanabe, Anna Takahashi and Aki Sekine \\ Niigata University of Pharmacy and Applied Life Sciences, Niigata, Japan
}

\begin{abstract}
The functionality of skin protective creams containing a film forming agent for enhancing barrier function was evaluated from the viewpoint of skin roughness prevention. The subjects were 15 healthy adults ( 7 females) without skin disorders ranging in age from 21-24 years (mean 22.1 years). Three sites on the medial side of the left forearm were used for measurements; an untreated control where no cream was applied, a small area applied with skin protective cream containing barrier ingredients, and a small area applied with skin protective cream containing no barrier ingredients. After repeated tape stripping for 20 times, transepidermal water loss (TEWL) and water content in the stratum corneum (SC) after applying skin protective cream were determined over time in each subject.

Barrier function index was calculated based on TEWL and water content in the SC, and used to evaluate the effects of the barrier ingredients. For the untreated control, the initial mean value obtained at 0 hours, and then 1,2 and 3 hours after skin protective cream application were $0.79,0.70,0.65$, and 0.62 , respectively. Those values for areas applied with cream containing no barrier ingredients were $0.42,0.44,0.43$, and 0.41 , respectively, while they were $0.39,0.42,0.39$, and 0.40 , respectively, for the areas applied with cream containing barrier ingredients. That later group showed the lowest values at each measurement time. Notably, high efficacy was seen for the cream containing barrier ingredients from 0 to 2 hours after application. These findings indicate that barrier function index is useful for evaluating the efficacy of skin protective creams.
\end{abstract}

\section{Introduction}

The stratum corneum (SC) has an extremely important role in preventing invasion by microorganisms and harmful substances into the body, while at the same time serves to retain moisture by preventing water evaporation from the body. Skin roughness is a collective term used to describe a disturbed surface condition that develops from a synergistic combination of a variety of factors, such as drying and inflammation. Once inflammation develops in the epidermis, water content in the SC is reduced. In skin formed under these conditions, the SC easily exfoliates as a mass, thus causing a reduction in barrier function. Furthermore, when skin roughness is left untreated, the risk of an infectious disease in the inflamed site is increased [1,2].

In the elderly, turnover of the epidermis is prolonged and skin metabolism is in a disturbed condition as a result of reduced cell division due to aging, making the skin of older individuals susceptible to pressure and friction as it hardens and becomes less elastic. Furthermore, atrophy of hair follicles and sebaceous glands result in a reduction in sweat and sebum secretion, as well as intercellular lipids, making it difficult to form a sebaceous membrane, which has a barrier function, causing dry skin $[3,4]$. In individuals with such fragile skin, the risk of developing pressure sores is also increased and recovery requires an extended period $[2,5]$.

Fundamental factors to maintain healthy skin are moisture and prevention from drying. Generally, skin-care cosmetic products are composed of water, oil, and moisturizing ingredients, and considered suitable for maintaining the balance of water, natural moisturizing factors, and lipids on the skin surface. Skin protective creams typically contain superfine-particle overcoat components such as silicon, which was developed as a preparation to protect the skin from various external factors, and is extensively used in the field of medical and nursing care [6].

For evaluating skin condition, measurements of high-frequency conductivity and transepidermal water loss (TEWL) are frequently utilized. The former is a technique used for determining water content in the SC according to the ease of electric flow in the layer, namely, changes in conductivity, which shows the effect of the base on water content in the SC $[7,8]$. TEWL is also termed transepidermal moisture loss and used as an index for evaluating skin barrier function, as it increases as that function decreases [3,4]. However, those measured values easily fluctuate, as they are affected by surrounding environmental factors such as temperature and humidity. Accordingly, it is essential to measure with care, including placing the subject in a room with constant temperature and humidity levels [9].

In the present study, the usefulness of barrier function index was examined based on the ratio of TEWL and water content in the SC of the skin after applying skin protective cream containing film formation ingredients.

*Correspondence to: Takahiro Kubota, Department of Biopharmaceutics, Faculty of Pharmaceutical Sciences, Niigata University of Pharmacy and Applied Life Sciences, F103a, 265-1 Higashijima, Akiha-ku, Niigata city 956-8603, Japan, Tel: +81-250-255288; E-mail: tkubota-tky@umin.net

Keywords: stratum corneum, skin protective cream, transepidermal water loss, water content

Received: June 18, 2019; Accepted: June 24, 2019; Published: June 27, 2019 


\section{Materials and methods}

\section{Test reagents and instruments}

Two kinds of skin protective creams were prepared, S-5132B, which contained barrier ingredients, and S-5132 without those. Both contained glycerin and PCA glyceryl isostearate as moisturizing ingredients, isopropyl myristate and squalene as emollients, retinol palmitate and ascorbyl tetraisopalmitate as skin conditioning agents, and stearic acid and simethicone as oily ingredients. Furthermore, poly-perfluoromethyl isopropyl ether, silsesquioxane, and myristoyl pullulan were added to S-5132B as film formation agents.

TEWL was determined using a VAPO SCAN AS-VT100RS, and water content in the SC with an ASA-MX100 thickness and moisture measuring system (Asahi Techno Lab, Tokyo). The measuring devices were used according to the instructions of the manufacturer. For tape-stripping, cellophane tape was used (CT-15S; Nichiban Co., Ltd., Tokyo).

\section{Subjects}

Fifteen healthy adults ( 7 females) without skin disorders and ranging from 21-24 years of age (mean 22.1 years) were used as subjects. Each was informed of the purpose and methods of the experiment, as well as handling of information obtained from the results, and provided consent verbally and in writing. The study protocol received approval from the Ethics Review Committee of Niigata University of Pharmacy and Applied Life Sciences (No. 004; July 31, 2014).

\section{Use of skin protective cream}

Three areas for performing measurements were established on the medial side of the left forearm of each subject, where tape-stripping was repeated 20 times. Those included an untreated control area with no cream applied, as well as an area applied with S-5132B cream (25 $\mu \mathrm{L})$ containing skin barrier ingredients and an area with S-5132 (25 $\mu \mathrm{L})$ containing no barrier ingredients. After tape-stripping, a water sorption-desorption test [10] was conducted, during which absorbent cotton soaked with purified water was placed on the area being examined for 30 seconds and removed, after which the skin was wiped with a paper towel.

The subjects were placed in the measuring room, kept at a temperature of $20-22^{\circ} \mathrm{C}$ with a humidity level ranging from $40-60 \%$, at 5 minutes before the start of the experiment under a calm environment [11]. The skin condition of each tested area was initially examined at 5 minutes after application of cream ( 5 minutes after starting the experiment in the control group) and the result was used as the initial value. Thereafter, measurements were conducted at 1,2, and 3 hours after the initial value was obtained. Measurements were conducted 5 times at each time point. The maximum and minimum values were excluded, and the mean of the values of the remaining 3 spots was used as the measurement.

\section{Skin condition and barrier function index determination}

TEWL water loss and water content in the SC were determined. The measurements were repeated 5 times, with the mean value after excluding the maximum and minimum used. Moreover, for comparing the barrier function efficacy in an objective manner, barrier function index was calculated using the following formula.

$$
\text { Barrier function index }=\frac{T E W L\left(\mathrm{~g} / \mathrm{hm}^{2}\right)}{\text { Water content }(\mu S)}
$$

\section{Statistical analysis}

For determining the difference between mean values of the 2 groups, a paired $t$ test was used, with the level of significance set as $5 \%$. Bellcurve for Excel (Social Survey Research Information Co., Ltd.) was used for the calculations.

\section{Results}

For the 15 subjects, TEWL on the medial side of the left forearm at 5 minutes after the start of the experiment or application of cream, used as the initial value, was $13.6 \pm 2.4 \mathrm{~g} / \mathrm{hm}^{2}$ (mean \pm standard deviation) for the untreated area, $14.8 \pm 1.8 \mathrm{~g} / \mathrm{hm}^{2}$ for the S-5132 area, and 10.3 $\pm 1.2 \mathrm{~g} / \mathrm{hm}^{2}$ for the S-5132B area ( $p=0.05$ vs. untreated, $p=0.0003$ vs. S-5132). Those values at 1 hour after application were 11.2 $\pm 1.9,10.0 \pm$ 1.4 , and $8.4 \pm 0.9 \mathrm{~g} / \mathrm{hm}^{2}$, respectively ( $p=0.05$ vs. untreated, $p=0.05$ vs. S-5132), at 2 hours after application were $10.6 \pm 1.8,9.7 \pm 1.4$, and $7.8 \pm$ $0.6 \mathrm{~g} / \mathrm{hm}^{2}$ respectively, and at 3 hours after application were $10.4 \pm 1.8$, $9.4 \pm 1.4$, and $7.8 \pm 0.7 \mathrm{~g} / \mathrm{hm}^{2}$, respectively (Figure 1).

Regarding water content in the SC on the medial side of the left forearm at 5 minutes after beginning the experiment, the initial value for the untreated area was $17.3 \pm 2.3 \mu \mathrm{S}$, while that for $\mathrm{S}-5132$ was $34.9 \pm$ $2.3 \mu \mathrm{S}$ ( $p<0.01$ vs. untreated) and for S-5132B was $26.7 \pm 1.6 \mu \mathrm{S}(p=0.001$ vs. S-5132). At the 1 hour time point, those were $16.1 \pm 2.4 \mu \mathrm{S}, 22.5 \pm$ $1.9 \mu \mathrm{S}$ ( $p<0.01$ vs. untreated), and $19.6 \pm 0.8 \mu \mathrm{S}$, respectively, $16.3 \pm 2.1$ $\mu \mathrm{S}, 22.4 \pm 1.6 \mu \mathrm{S}$ ( $p<0.01$ vs. untreated), and $19.8 \pm 0.7 \mu \mathrm{S}$, respectively, after 2 hours, and $16.7 \pm 2.2 \mu \mathrm{S}, 22.6 \pm 1.9 \mu \mathrm{S}$ ( $p<0.01$ vs. untreated), and $19.7 \pm 0.9 \mu \mathrm{S}$, respectively, after 3 hours (Figure 2 ).

\section{Discussion}

Barrier function efficacy in the presence or absence of barrier ingredients was examined using a barrier function index, which was calculated based on water content in the SC and TEWL. For the untreated skin area, the initial value, and values obtained a 1,2 , and 3 hours were $0.79,0.70,0.65$, and 0.62 (arbitrary units), respectively, while those were $0.42,0.44,0.43$, and 0.41 , respectively, for the S-5132 area, and $0.39,0.42,0.39$, and 0.40 , respectively, for the S-5132B area, with the latter values the lowest at all time points (Figure 3 ). Both the S-5132 and S-5132B creams were confirmed to be effective for enhancing barrier function, with S-5132B notably exhibiting a superior effect up to 2 hours after application. On the other hand, the difference in barrier function index value between the creams was only slight at 3 hours, thus we speculated that the barrier effect of S-5132B may remain for 2-3 hours after application.

Both the S-5132 and S-5132B creams contained glycerin and PCA glyceryl isostearate, widely used for moisturizing and anti-inflammation effects, as well as the commonly used stearyl glycyrrhetinate, tocopherol acetate, and retinol palmitate. However, film formation ingredients were also added to the S-5132 cream, including poly-perfluoromethyl isopropyl ether of the fluorine series, silsesquioxane of the silicon series, and myristoyl pullulan, a water-resistant oil-soluble natural polysaccharide, which are typically used to protect skin from external factors such as hand washing and use of rubbing alcohol. We found that application of S-5132B caused a lower water content in the SC, likely because of changes in conductivity or inhibition of permeation from the effects of the film formation and moisturizing ingredients (Figure 2). Additionally, it is considered that the barrier function shown by the S-5132B cream might have been enhanced more by suppression of TEWL from the SC because of its film formation quality (Figures 1 and 3). 


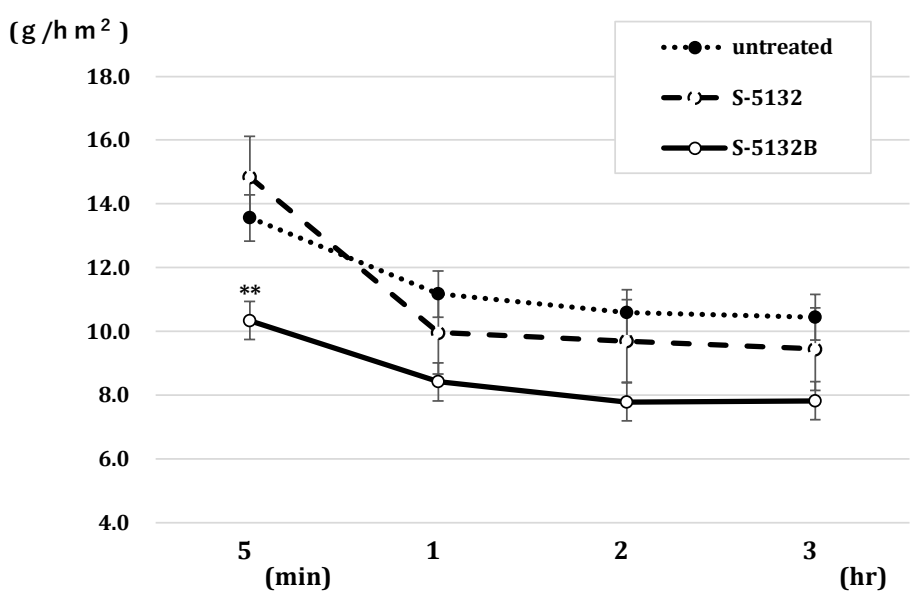

Figure 1. Changes in TEWL over time under the tested conditions

Values are shown as the mean \pm standard deviation $(\mathrm{n}=15) .{ }^{* *} p<0.01, \mathrm{~S}-5132$ vs. S-5132B

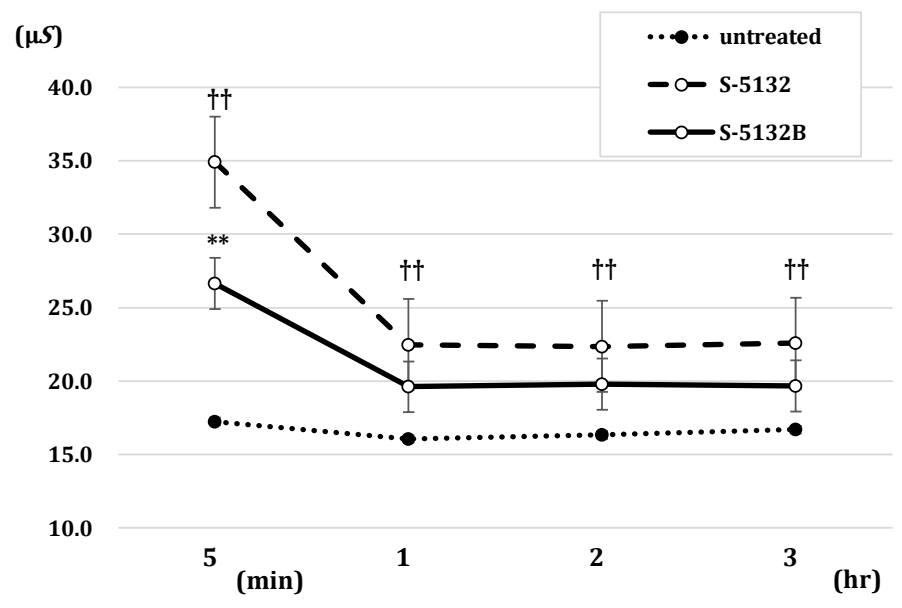

Figure 2. Changes in water content in the SC over time under the tested conditions

Values are shown as the mean \pm standard deviation $(\mathrm{n}=15) .{ }^{* *} p<0.01, \mathrm{~S}-5132$ vs. S-5132B; $\dagger \dagger p<0.01$, untreated vs. S-5132

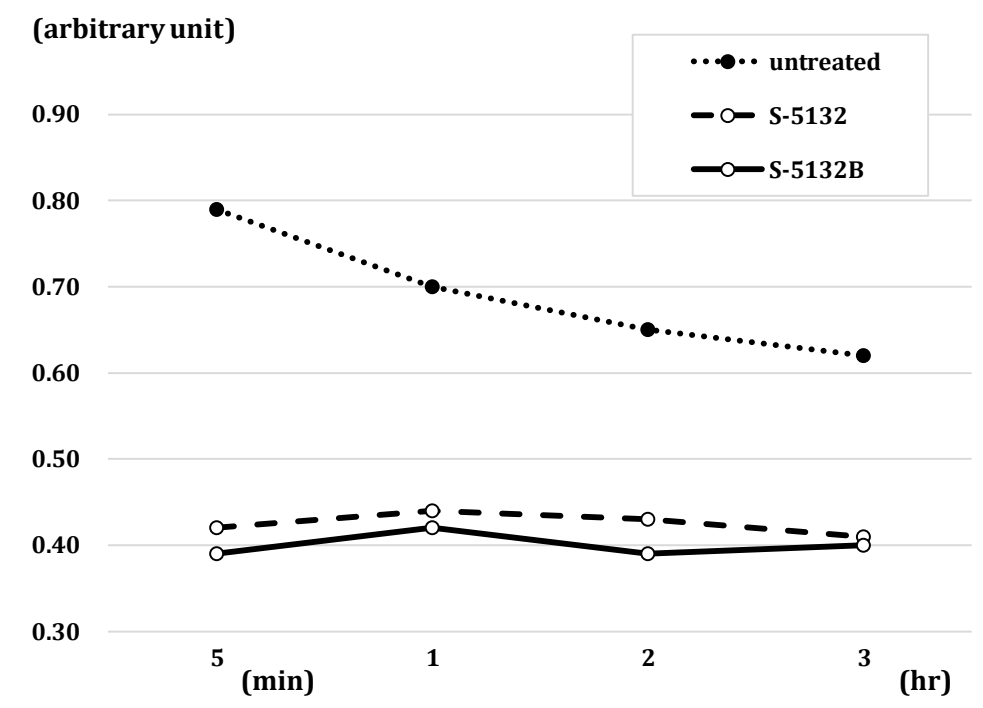

Figure 3. Changes in barrier function index over time in areas applied with creams with and without barrier ingredients 
The present results confirmed superior performance for TEWL and water content in the SC by a film formation capable cream (S-5132B) as compared to a cream (S-5132) without those related ingredients. In addition, use of a barrier function index that includes both values for calculation was shown to be effective for comparing the efficacy of skin protective creams, with higher efficacy shown by a lower value.

\section{Conflicts of interest statement}

The authors have no conflicts of interest to declare in association with this study.

\section{References}

1. Elias PM (1988) Structure and function of the stratum corneum permeability barrier. Drug Dev Res 13: 97-105.

2. Watanabe M, Tagami H, Horii I, Takahashi M, Kligman AM (1991) Functional analyses of the superficial stratum corneum in atopic xerosis. Arch Dermatol 127: $1689-1692$

3. Kikuchi K, Kobayashi H, Hirao T, Ito A, Takahashi H, et al. (2003) Improvement of mild inflammatory changes of the facial skin induced by winter environment with daily applications of a moisturizing cream. A half-side test of biophysical skin parameters, cytokine expression pattern and the formation of cornified envelope. Dermatology 207: 269-275.
4. Kikuchi K, Tagami H (2008) Japanese Cosmetic Scientist Task Force for Skin Care of Atopic Dermatitis. Noninvasive biophysical assessments of the efficacy of a moisturizing cosmetic cream base for patients with atopic dermatitis during different seasons. Br J Dermatol 158: 969-978.

5. Hara M, Kikuchi K, Watanabe M, Denda M, Koyama J, et al. (1993) Senile xerosis: functional, morphological and biochemical studies. J Geriatr Dermatol 1: 111-120.

6. Kubota T (2012) Evaluation of skin surface hydration state and barrier function of stratum corneum of dorsa of hands and heels treated with PROTECT X2 skin protective cream. Drug Discov Ther 6: 157-162.

7. Obata M, Tagami H (1989) Electrical determination of water content and concentration profile in a simulation model of in vivo stratum corneum. J Invest Dermatol 92: 854-859.

8. Egawa M, Hirao T, Takahashi M (2007) In vivo estimation of stratum corneum thickness from water concentration profiles obtained with Raman spectroscopy. Acta Derm Venereol 87: 4-8.

9. Tagami H, Ohi M, Iwatsuki K, Kanamaru Y, Yamada M, et al. (1980) Evaluation of the skin surface hydration in vivo by electrical measurement. J Invest Dermatol 75: 500-507.

10. Tagami H, Kanamaru Y, Inoue K, Suehisa S, Inoue F, et al. (1982) Water sorptiondesorption test of the skin in vivo for functional assessment of the stratum corneum. $J$ Invest Dermatol 78: 425-428.

11. Berardesca E (1997) European Group for Efficacy Measurements on Cosmetics and Other Topical Products (EEMCO). EEMCO guidance for the assessment of stratum corneum hydration: electrical methods. Skin Res Technol 3: 126-132.

Copyright: $\odot 2019$ Kubota T. This is an open-access article distributed under the terms of the Creative Commons Attribution License, which permits unrestricted use, distribution, and reproduction in any medium, provided the original author and source are credited. 